\title{
Significance of Poisson distribution theory in analysing the interaction between human spermatozoa and zona- free hamster oocytes*
}

\author{
R. J. Aitken and R. A. Elton $\dagger$ \\ $M R C$ Unit of Reproductive Biology and $\nmid$ Medical Computing and Statistics Unit, University of \\ Edinburgh, U.K.
}

\begin{abstract}
Summary. The value of Poisson distribution theory in describing and predicting the nature of sperm-egg interaction in vitro has been investigated using an interspecific invitro fertilization system, incorporating zona-free hamster oocytes and human spermatozoa. The frequency distribution of polyspermic oocyte penetrations in 72 experiments exhibited good agreement with the Poisson distribution at all levels of fertilization, indicating that each oocyte must be of equal penetrability and that there can be no block to polyspermy in this interspecific system. Poisson distribution theory also accurately described the relationship between oocyte penetration and sperm motility in 50 out of 54 separate experiments spread across 10 serial dilution curves. For each dilution series the shape of the fitted curve was fixed but its location along the $x$ axis varied from donor to donor. The fixed nature of the relationship between sperm motility and egg penetration enables the results of such in-vitro fertilization experiments to be corrected for the number of motile spermatozoa in the incubation media. On the basis of these findings a protocol is described for assessing the results of the zona-free hamster oocyte penetration assay, which involves analysis of the degree of polyspermy followed by the application of Poisson distribution theory to correct the results to a standard concentration of motile spermatozoa. Changes in the penetrating ability of human spermatozoa after vasectomy and characterization of the degree of inter-ejaculate variation in penetrating potential are two clinical examples of such analyses given in the text. The statistical methods described in this paper should also be of general relevance to the study of fertilization mechanisms, in providing a rationale by which to analyse the quantitative nature of sperm-egg interaction in vitro.
\end{abstract}

\section{Introduction}

The assessment of human sperm function has recently been facilitated by the development of an interspecific in-vitro fertilization system, involving the fusion of human spermatozoa with the vitelline membrane of zona-free hamster oocytes (Yanagimachi, Yanagimachi \& Rogers, 1976). The ability of human spermatozoa to penetrate hamster oocytes has been shown to vary according to the fertility status of the sperm donor, and significantly lower levels of fertilization are encountered in conditions such as idiopathic infertility, oligozoospermia and varicocoele, than in the normal fertile population (Rogers et al., 1979; Rogers, McCarville, Mygatt, Soderdahk \& Hale, 1980; Stenchever et al., 1982; Aitken et al., 1982a, b, c). Correlations have also been observed between hamster egg penetration and the duration of infertility (Cohen, Weber, Van der Vijver \& Zeilmaker, 1982), while the prognostic value of this system has been confirmed in a prospective

* Address for reprints: Dr R. J. Aitken, MRC Unit of Reproductive Biology, Centre for Reproductive Biology, 37 Chalmers Street, Edinburgh EH3 9EW, U.K. 
study of patients exhibiting infertility of unknown aetiology (Aitken, Best, Warner \& Templeton, 1984).

Despite the putative importance of this in-vitro fertilization system, uncertainty has arisen with respect to the interpretation of the procedure. In particular, a rationale has not yet been developed for incorporating factors such as the degree of polyspermy or the modulating influence of sperm motility (Binor, Sokoloski \& Wolf, 1980; Aitken et al., 1982a; Aitken, Liu, Best \& Richardson, 1983a) into the expression of the results. Underlying these deficiencies is the absence of any critical analyses of the quantitative nature of sperm-egg interaction during in-vitro fertilization. In this study we have examined the nature of this interaction and its accordance with Poisson distribution theory. The results are of general significance in providing a logical foundation on which to base the analysis of in-vitro experiments concerning the mechanisms of sperm-egg interaction during fertilization.

\section{Materials and Methods}

\section{Semen preparation and analysis}

Semen samples were obtained after abstinence for at least $48 \mathrm{~h}$ by masturbation into sterile plastic containers. After allowing at least $30 \mathrm{~min}$ for liquefaction to occur, a conventional semen analysis was carried out on each sample to determine sperm motility, morphology, total count and concentration, using the procedures laid down by the World Health Organization (1980). Unless otherwise stated all donors were normal healthy males exhibiting a normal semen profile ( $>50 \%$ motility, $>40 \%$ normal morphology, $>20 \times 10^{6} / \mathrm{ml}$ ). In a group of 12 normal fertile males, semen specimens were obtained during the month before and within 12 days after, a vasectomy operation (Richardson, Aitken \& Loudon, 1984).

The ability of the spermatozoa to penetrate zona-free hamster oocytes was assessed at a fixed concentration of $10 \times 10^{6}$ cells $/ \mathrm{ml}$ using the protocol described by Aitken, Wang, Liu, Best \& Richardson (1983c). Assessments of sperm motility were carried out at $\times 250$ with the aid of an eyepiece graticule. These determinations were performed at the end of a 3-h period of capacitation in hyperosmotic medium immediately before the introduction of the zona-free hamster oocytes.

\section{The Poisson model}

The basic theoretical model proposed and tested in this paper is that the outcome of the hamster egg penetration test in terms of the number and distribution of oocyte penetrations is the result of random variation in the number and distribution of sperm-egg collisions.

The first tenet of this model is that the spermatozoa are randomly distributed throughout the incubation medium and hence the probability of a particular motile spermatozoon occurring in a given volume of the sample does not depend on how many other spermatozoa are in that volume. The existence of such a randomly mixed distribution of particles in the medium leads to the prediction that the number of spermatozoa in any specified volume, $y$, of the sample should follow a Poisson distribution:

$$
P_{1}(r, x, y)=\left[\exp (-x y)(x y)^{r}\right] / r !
$$

where $P_{1}(r, x, y)$ is the probability that a volume, $y$, contains exactly $r$ spermatozoa and $x$ is the global average concentration of spermatozoa per unit volume.

A second tenet, that each oocyte is of equal penetrability, leads to the expectation that the number of spermatozoa penetrating each egg will also have a Poisson distribution:

$$
P_{2}(r, x, \theta)=\left[\exp (-\theta x)(\theta x)^{r}\right] / r !
$$

where $P_{2}(r, x, \theta)$ is the probability that an egg will be penetrated by exactly $r$ spermatozoa, $x$ is the concentration of spermatozoa per unit volume as before, and $\theta$ is a constant factor reflecting the 
proportion of spermatozoa per unit volume that are sufficiently close to an oocyte to make contact with the vitelline membrane and initiate fusion. The factor $\theta$ thus subsumes all considerations of sperm quality, kinetics and experimental conditions into a single parameter.

\section{Experimental examination of the Poisson model}

The fit of this model to real data has been examined in 3 different ways.

Comparison of $\%$ penetration (p) and mean number of penetrations per oocyte $(\mathrm{m})$. One method is to compare the mean number, $m$, of spermatozoa penetrating each oocyte, with the proportion, $p$, of oocytes penetrated. According to the Poisson model, $p$ and $m$ should be related by the formula:

$$
p=1-\exp (-m)
$$

and an informal test of the model can then be obtained by seeing how closely the observed and predicted value for $p$ correspond.

Examination of the frequency distribution of sperm penetrations. A more detailed assessment of the Poisson model can be obtained by comparing the observed frequency distribution of sperm penetrations per oocyte with the expected frequencies based on the mean value, $m$. The correspondence of observed values to the Poisson distribution can then be tested by the statistic

$$
X^{2}=(n-1) v / m
$$

where $v$ is the sample variance of the number of the spermatozoa per oocyte, $n$ is the number of oocytes and $X^{2}$ is tested against $\chi^{2}$ with $n-1$ degrees of freedom.

Dilution series. The validity of the Poisson model can also be tested by analysing the penetration rates $(\%$ oocytes penetrated) observed with individual sperm suspensions examined over a range of dilutions. According to the model, the penetration $p$ at any given concentration of motile spermatozoa, $x$, should be predicted by the formula:

$$
p=1-\exp (-\theta x)
$$

where $\theta$ is an unknown constant, reflecting the experimental conditions and the proportion of spermatozoa in the sample that are capable of fusion with the vitelline membrane. If a series of values $\left[\left(p_{\mathrm{i}}, x_{\mathrm{i}}\right), \mathrm{i}=1, \ldots, \mathrm{k}\right]$ are available, where $p_{\mathrm{i}}=r_{\mathrm{i}} / n_{\mathrm{i}}$ is based on an experiment with $n_{\mathrm{i}}$ oocytes, then $r_{\mathrm{i}}$ should have a binomial distribution with probability $1-\exp \left(-\theta x_{\mathrm{i}}\right)$. Since the model can be expressed as

$$
\log [-\log (1-p)]=\log (\theta)+\log (x)
$$

(where natural logarithms are used), the theory of generalized linear models (Nelder \& Wedderburn, 1972) can be applied. This allows the data to be fitted by the statistical package GLIM using a binomial error, a complementary log-log link and an offset of unity for $\log (x)$ (GLIM, 1978; Wetherill, 1981). This yields a statistic termed 'the deviance' which measures the goodness-of-fit to the model and can be tested for significance by comparison to $\chi^{2}$ with $\mathrm{k}-1$ degrees of freedom. A simpler and more efficient analysis can be done if data on the numbers of spermatozoa penetrating each oocyte are also available for each experiment in the dilution series. Suppose that the experiment indexed by $i$ gives a mean of $m_{\mathrm{i}}$ spermatozoa per oocyte. The theory then predicts that $n_{\mathrm{i}} m_{\mathrm{i}}$ should have a Poisson distribution, with mean value $\theta n_{\mathrm{ixi}}$, and the fit can be tested by a $\chi^{2}$ test comparing the $\mathrm{k}$ observed frequencies $\left[n_{\mathrm{i}} m_{\mathrm{i}}, i=1, \ldots, \mathrm{k}\right]$ with expected frequencies $\left[\operatorname{tn}_{\mathrm{i}} x_{\mathrm{j}}, \mathrm{i}=1, \ldots, \mathrm{k}\right]$ where

$$
t=s\left(n_{\mathrm{i}} m_{\mathrm{i}}\right) / s\left(n_{\mathrm{i}} x_{\mathrm{i}}\right)
$$

and $s$ denotes summation over the $\mathrm{k}$ experiments. The $\chi^{2}$ statistic

$$
X^{2}=s\left[\left(n_{\mathrm{i}} m_{\mathrm{i}}-t n_{\mathrm{i}} x_{\mathrm{i}}\right)^{2} /\left(t n_{\mathrm{i}} x_{\mathrm{i}}\right)\right]
$$


can then be examined with $\mathbf{k}-1$ degrees of freedom. The statistic $t$ is an estimate of $\theta$ combining the information from all the experiments.

The experiments reported in the following section examine the validity of the Poisson model for describing the outcome of the in-vitro fertilization procedure, using the 3 different methods of investigation cited above. The value of Poisson distribution theory in correcting in-vitro fertilization results for any desired concentration of motile spermatozoa and the practical application of this facility for studying the effects of vasectomy and inter-ejaculate variations in sperm quality are subsequently examined.

\section{Results}

Comparison of $\%$ penetration $(\mathrm{p})$ and mean number of penetrations/oocyte $(\mathrm{m})$

The results of 72 separate assessments of hamster oocyte penetration are presented in Text-fig. 1 , which plots $\mathrm{p}$ against $m$ for each experiment, and incorporates the theoretical curve derived from Poisson distribution theory. As the proportion of oocytes penetrated increased, there was a concomitant rise in the degree of polyspermy attained, which continued even after $100 \%$ penetration had been reached. In most experiments the data exhibited a close fit to the Poisson distribution curve, with an equal distribution of points above and below the line.

\section{Examination of the frequency distribution of sperm penetrations}

The frequency distribution of oocyte penetrations observed within each experiment was subsequently analysed to determine the goodness-of-fit with the expected frequencies, based on the mean number of spermatozoa per oocyte, $m$. Examples of this analysis, from the total group of 72

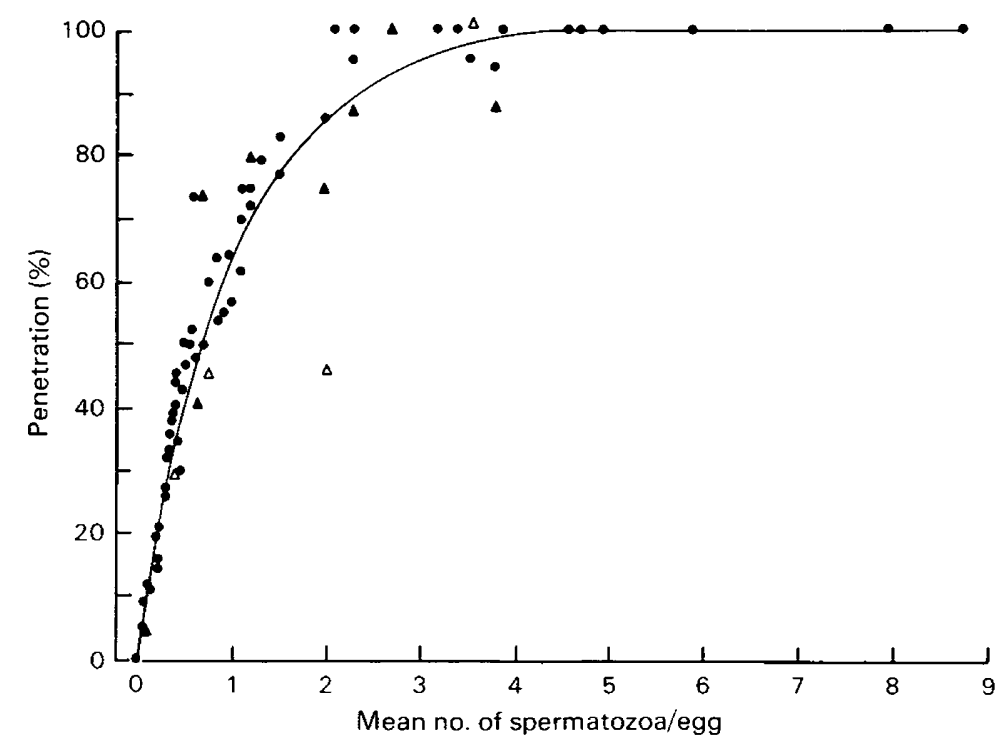

Text-fig. 1. Relationship between the mean number of spermatozoa per egg and \% penetration: $\boldsymbol{\Delta}$ indicates experiments in which the frequency distribution of egg-penetrations deviated significantly from expected levels; $\Delta$ deviant experiments in which cellular contamination of the ejaculate or marked sperm agglutination were observed. Solid line describes the relationship predicted by Poisson distribution theory. 
assessments described in Text-fig. 1, are given in Text-fig. 2 in which a close approximation of observed and expected frequency distribution is apparent. The $\chi^{2}$ goodness-of-fit test indicated that out of the 72 experiments performed, $60(83 \%)$ yielded results which were not significantly different from the theoretically expected values. The 12 experiments in which significant deviation from a Poisson distribution was observed are indicated on Text-fig. 1. In 4 of these samples, cellular contamination (pyospermia) of the semen was noted which in 2 cases was associated with agglutination of the spermatozoa.

\section{Dilution series}

Text-figure 3 shows the observed penetration rates and the fitted curves for 10 serial dilution experiments. The dilution is shown on a logarithmic scale for which the fitted curves are of fixed shape but variable location; the latter, in turn, depends on the value of $\theta$, which can be estimated from the experimental data. Out of 10 dilution series only 2 contained points deviating significantly from the Poisson model. For these 2 deviant series, discrepant points were identified by examining the standardized residuals, i.e. the ratio of the deviation of each point from the curve to the residual standard deviation. Those points for which this value exceeds 2 are indicated by arrows in Textfig. 3 . The results indicate that in only 4 of the 54 individual incubations illustrated in Text-fig. 3 were the data inconsistent with the Poisson model.

Analysis of the mean number of spermatozoa penetrating each oocyte for the experiments depicted in Text-fig. 3 also indicated good accordance with the Poisson model, with the same 2 dilution series being identified as discrepant (Table 1).

\section{Theoretical extrapolation of in-vitro fertilization results}

The apparent accordance between the behaviour of spermatozoa and oocytes during in-vitro fertilization and the Poisson distribution model permits the theoretical extrapolation of the results

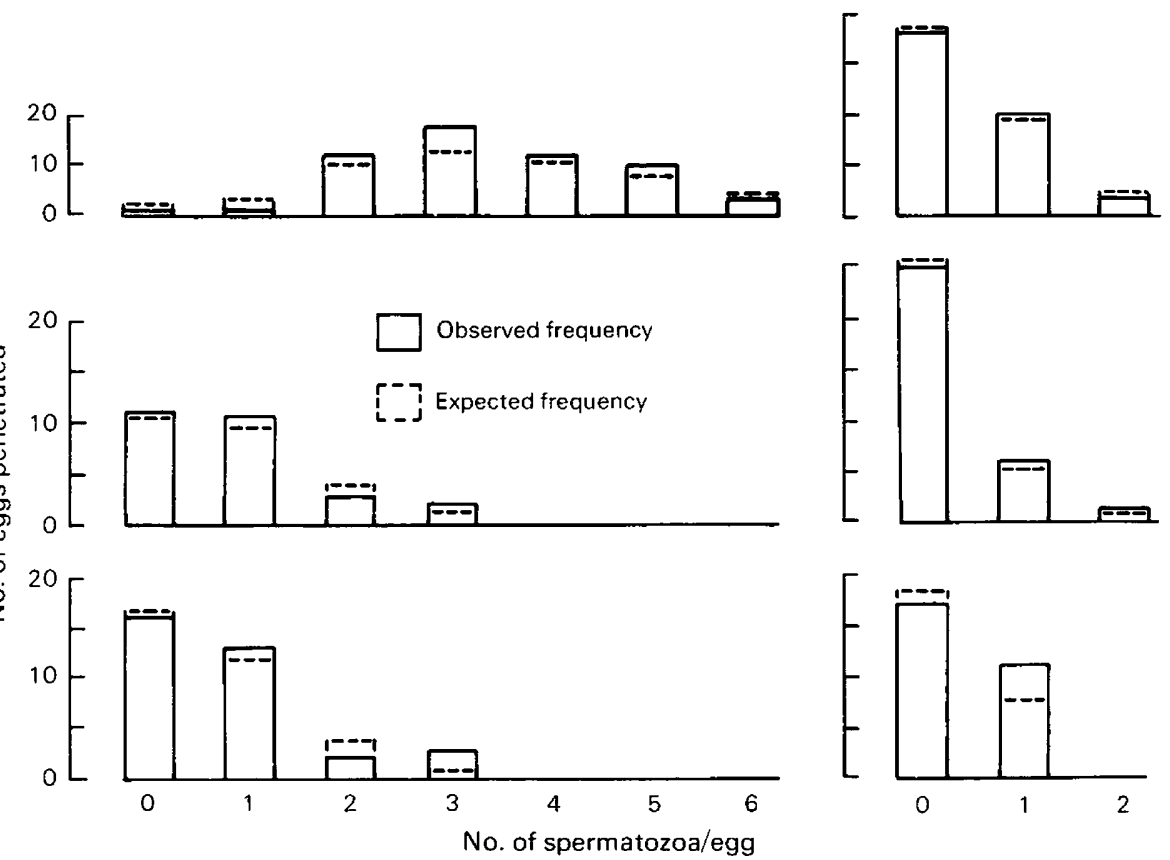

Text-fig. 2. Observed (- $(-$ and expected (---) frequency distribution of sperm penetrations. Each panel represents an experiment on a separate semen sample from the total group of 72 assessments indicated in Text-fig. 1. 

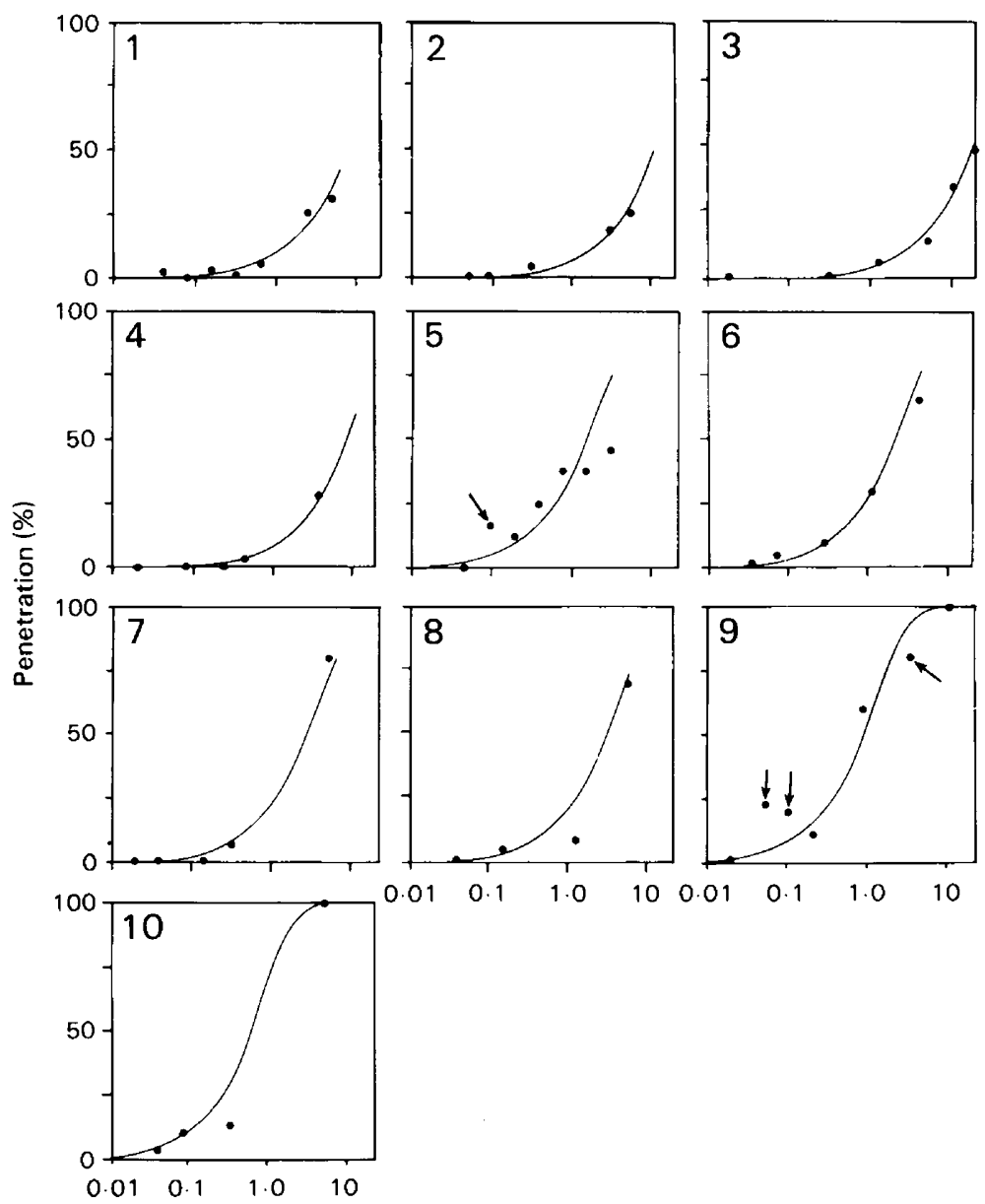

Motile sperm conc./ml

Text-fig. 3. Serial dilution curves for 10 specimens. Solid line describes the relationship predicted by Poisson distribution theory. Arrows indicate deviant results

Table 1. Statistical analysis of dilution series data: deviance statistic using $p$ and $\chi^{2}$ statistic using $m$ (the degrees of freedom for the two tests differ because categories with low expected frequency have been merged in the $\chi^{2}$ test)

\begin{tabular}{|c|c|c|c|c|c|c|c|}
\hline Series* & $\begin{array}{l}\text { No. of } \\
\text { exps }\end{array}$ & Deviance & d.f. & Significance & $\chi^{2}$ & d.f. & Significance \\
\hline 1 & 7 & $5 \cdot 26$ & 6 & NS & 0.06 & 2 & NS \\
\hline 2 & 5 & 1.07 & 4 & NS & 0.25 & 1 & NS \\
\hline 3 & 6 & 1.80 & 5 & NS & 0.35 & 1 & NS \\
\hline 4 & 5 & 1.43 & 4 & NS & $0 \cdot 17$ & 1 & NS \\
\hline 5 & 7 & 13.59 & 6 & $P<0.05$ & 11.49 & 3 & $P<0.01$ \\
\hline 6 & 5 & $2 \cdot 37$ & 4 & NS & 2.55 & 2 & NS \\
\hline 7 & 5 & $2 \cdot 62$ & 4 & NS & 0.57 & 1 & NS \\
\hline 8 & 4 & $3 \cdot 51$ & 3 & NS & $2 \cdot 54$ & 1 & NS \\
\hline 9 & 6 & 24.99 & 5 & $P<0.001$ & $52 \cdot 14$ & 4 & $P<0.001$ \\
\hline 10 & 4 & $3 \cdot 32$ & 3 & NS & $2 \cdot 67$ & 2 & NS \\
\hline
\end{tabular}

* See Text-fig. 3. 
of a single experiment to that which would have been observed at any other concentration of motile spermatozoa, within the limits indicated below. Hence if a proportion $p_{1}$ of eggs are penetrated at a concentration $x_{1}$ of motile spermatozoa per ml, the formula for estimating $p_{2}$, when $0<p_{1}<1$, is:

$$
p_{2}=1-\left(1-p_{1}\right)^{r}
$$

where $r=x_{2} / x_{1}$, representing the ratio of the two concentrations. Confidence limits for the estimate $p_{2}$ can be obtained by applying this formula to the corresponding limits for $p_{1}$ based on the binomial distribution (see Documenta Geigy, 1968, pp. 85-103). This can be done even when $p_{1}$ is 0 or 1, giving upper or lower limits respectively for the percentage penetration at any given concentration. For example, suppose that 13 out of 50 eggs $(26 \%$, with $95 \%$ confidence limits of 14.6 and $40.3 \%$ are penetrated at a concentration of $1.33 \times 10^{6}$ motile spermatozoa $/ \mathrm{ml}$, and the penetration is to be estimated for a theoretical motile concentration of $5 \times 10^{6} / \mathrm{ml}$. Then substituting into the above formula gives:

$$
p_{2}=1-(1-0 \cdot 26)^{3 \cdot 76}
$$

yielding a $p_{2}$ value of $0.678(67.8 \%)$ with $95 \%$ confidence limits of 0.448 and $0.856(44.8-85.6 \%)$. Hence with the aid of such calculations the results of this in-vitro fertilization procedure may be standardized for any fixed concentration of motile spermatozoa.

Extrapolation of penetration to a standardized sperm concentration is only limited by the fact that samples exhibiting penetration rates of $100 \%$ are not suitable for analysis. This problem is solved if the mean number, $m_{1}$, of spermatozoa per egg is known at the experimental concentration $x_{1}$. The degree of polyspermy, $m_{2}$, which would be observed at the theoretical sperm density $x_{2}$, can then be calculated as $m_{2}=r m_{1}$ where $r=x_{2} / x_{1}$. The penetration rate at $x_{2}$ can then be calculated from the formula

$$
p_{2}=1-\exp \left(-r m_{1}\right)
$$

Confidence limits for $p_{2}$ can be obtained from tables (Documenta Geigy, 1968, p. 107) by using the fact that $n m_{1}$, the total number of penetrating spermatozoa (where $n$ is the number of oocytes in the experiment) has a Poisson distribution. For example, suppose that for the experiment described earlier, with 50 oocytes and a penetration rate of $26 \%$, the mean number of spermatozoa per egg had been 0.30 and the value for $n m_{1}$ had therefore been 15 . The Poisson distribution tables give $95 \%$ confidence limits of $8 \cdot 40-24.7$ for $n m_{1}$ and when these values are substituted into the above formula, the confidence limits for $p_{2}$ are given by $1-\mathrm{e}^{-3.76 \times 0.168}$ and $1-\mathrm{e}^{-3.76 \times 0.494}$, yielding values of $0.468(46.8 \%)$ and $0.844(84.4 \%)$ respectively. These confidence limits and the actual predicted $p_{2}$ value, calculated from $m_{1}=0.3$, of $0.676(67.6 \%)$ are very similar to the values previously calculated on the basis of $p_{1}$ alone, i.e. $67 \cdot 8 \%(44 \cdot 5-85 \cdot 6 \%)$. This will be the case when $m_{1}$ is small but when $m_{1}$ is larger (e.g. $>1$ ) methods based on $m_{1}$ rather than $p_{1}$ can be expected to give considerably narrower limits. Calculations based on the mean number of spermatozoa per egg rather than penetration rate also have the advantage of being able to give two-sided limits even when the latter reaches $100 \%$.

\section{Application of Poisson distribution theory}

The practical application of predictions based on Poisson distribution theory can be illustrated by considering the in-vitro fertilization performance of human spermatozoa recovered from successive ejaculates or before and after vasectomy. In both cases the interest is in whether the difference in penetrating ability between samples can be explained purely on the basis of variations in sperm motility, i.e. in accordance with the Poisson model.

The simplest statistic for measuring the excess variation in such series is the ratio of the $\chi^{2}$ statistic to its degree of freedom, which should be approximately unity if there is no extra variation. In Text-fig. 4 this statistic is plotted for the 10 dilution series experiments illustrated in Text-fig. 3, 


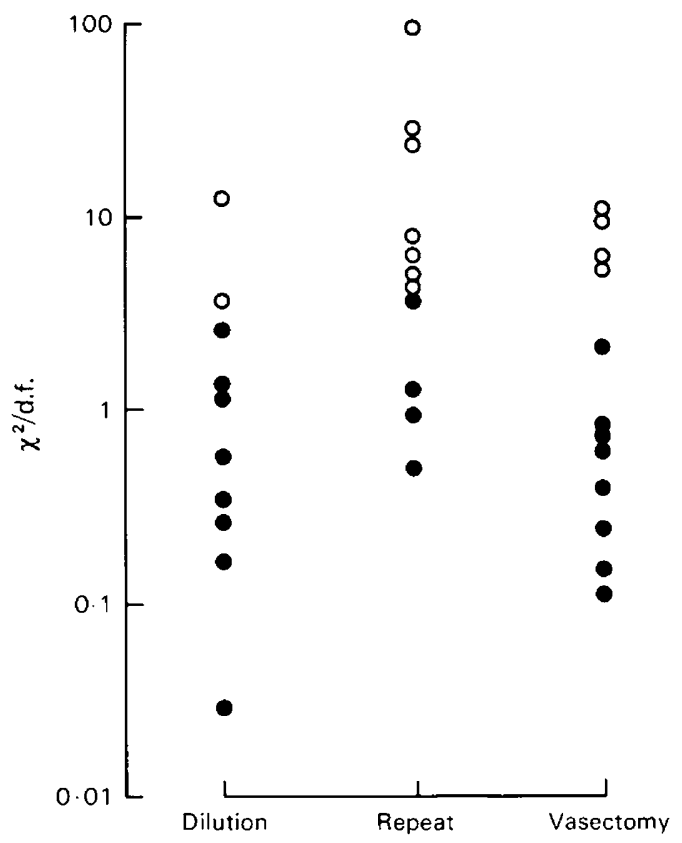

Text-fig. 4. Excess variation over model prediction as measured by the ratio of the $\chi^{2}$ statistic to its degrees of freedom for 10 dilution series (see Text-fig. 3), 12 repeat sample series and 12 men examined both before and after vasectomy. Significant series are shown as open circles.

for repeat ejaculates from 12 donors (an average of $3 \cdot 2$ ejaculates per donor with a range of 2-6 ejaculates) and for 12 patients sampled before and within 12 days of vasectomy. There was a significant difference between the dilution series experiments and the variation observed for successive ejaculates (Wilcoxon rank sum test, $P<0.01$ ) indicating that, while the degree of intraassay variation is low, the penetrating potential of human spermatozoa does exhibit significant interejaculate variation, which is independent of any differences in motility. When Poisson distribution theory was used to correct the results of the vasectomy series for the large differences in post-capacitation sperm motility observed before $\left(4 \cdot 1 \pm 0.3 \times 10^{6}\right.$ motile spermatozoa/ml) and after $\left(1.8 \pm 0.4 \times 10^{6}\right.$ motile spermatozoa $\left./ \mathrm{ml}\right)$ vas ligation, the results appeared remarkably homogeneous (Text-fig. 4). Hence in $66.7 \%$ of tests the post-vasectomy decline in hamster oocyte penetration rates (from a pre-ligation value of $42.0 \pm 8.4 \%$ to a post-operative score of $11.3 \pm$ $4.9 \%$ ) could be accounted for by the concomitant decline in sperm motility.

\section{Discussion}

This study has indicated the importance of Poisson distribution theory in describing the quantitative nature of sperm-egg interaction during fertilization in vitro. The frequencydistribution of egg penetrations, the relationship between the proportion of oocytes penetrated and the degree of polyspermy and the influence of sperm motility on fertilization rates have all been found to conform to the Poisson distribution. In specific terms of the zona-free hamster egg penetration test, the existence and predictive power of such theoretical relationships will be of value in standardizing the results of the assay for differences in sperm motility and in providing a means by which the procedure might be quality controlled. 
In the past, attempts have been made to account for the modulating influence of motility on the outcome of this interspecific system by fixing the concentration of motile spermatozoa in the incubation medium to $0.3-0.4 \times 10^{7} / \mathrm{ml}$, irrespective of total sperm numbers. Hence in conditions such as asthenozoospermia, oligozoospermia or varicocoele, in which the proportion of motile spermatozoa is normally $<40 \%$, the desired sperm concentration has only been attained at the cost of large numbers of immotile cells and cellular debris in the incubation medium. Adjustment of motile sperm numbers at the beginning of an experiment also suffers from the disadvantage that it does not provide any means of accounting for changes in sperm motility which occur during the course of an incubation which in some conditions may be significant (Aitken et al., 1982b). In contrast, such changes can readily be assimilated through the retrospective application of Poisson distribution theory. Physical adjustment of motile sperm numbers may be facilitated by the use of layering procedures or density gradient centrifugation, to isolate the motile spermatozoa from a semen specimen. However, since such procedures are of reduced effectiveness in cases of asthenozoospermia and do not yield preparations which are uniformly motile, Poisson distribution theory will still be of significant value in standardizing the results for a fixed concentration of motile spermatozoa.

In the light of these considerations, it is recommended that the zona-free hamster egg penetration test is carried out at a fixed total sperm concentration of $10 \times 10^{6} / \mathrm{ml}$ (Rogers et al., 1979; Aitken et al., 1982a, b, c). The in-vitro fertilization results can then be assessed in terms of both the degree of polyspermy observed (mean number of spermatozoa per egg) and sperm motility (at the moment when the oocytes are introduced) and these figures incorporated in the Poisson expression $p_{2}=1-\exp \left(-r m_{1}\right)$ in order to obtain a penetration value $(\%$ penetration or mean number of spermatozoa/egg) which has been standardized for a fixed concentration of motile spermatozoa, say $5 \times 10^{6} / \mathrm{ml}$. The mean number of spermatozoa per egg is the most logical criterion to select because it not only incorporates all of the information obtained by examination of the oocytes, but also bears a direct relationship with sperm motility at all levels of fertilization; a factor which becomes particularly important as the percentage of oocytes penetrated approaches $100 \%$. These findings indicate that there can be no block to polyspermic fertilization in this model; a conclusion which was also reached by Binor et al. (1982) on the basis of repeated insemination experiments.

Another major problem in the clinical application of this interspecific in-vitro fertilization procedure relates to the absence of any quality-control criteria by which to assess the reliability of assays established in independent laboratories. The accordance between the results obtained in this study and Poisson distribution theory provides a means of achieving such quality control. The frequency distribution of polyspermic penetrations within each experiment, should, by their degree of conformity to Poisson distribution theory, indicate the consistency of the oocyte preparations, since all oocytes have been shown in this study to exhibit an equal susceptibility to penetration. Care should be taken to exclude pyospermic or agglutinated samples from such analyses since these conditions were found to prevent spermatozoa from exhibiting the independent behaviour required by Poisson distribution theory.

Another important component in the development of quality control criteria for this system would be to perform serial-dilution experiments and to examine the relationship between the results obtained and the Poisson expressions described in this paper. Good agreement in this context would indicate that the experimental conditions (subsumed into $\theta$ and covering such conditions as light, temperature, humidity, accuracy and consistency of the sperm preparations) are being adequately controlled and that the degree of intra-assay variation is not significant. Although hyperosmotic media were used to accelerate sperm capacitation in this study, serial dilution curves constructed using samples capacitated under normo-osmotic conditions or exposed to the cation ionophore, A23187, also exhibited the anticipated agreement with Poisson distribution theory (R. J. Aitken, F. Best \& R. Elton, unpublished observations). In principle, as long as the acrosomereacted spermatozoa are randomly distributed in the sperm population, agreement with the Poisson 
model should be obtained regardless of the composition of the incubation media or differences in methodological detail.

Assessment of interassay variation is not so readily determined since the present results indicate that ejaculates from the same donor exhibit significant variation, even when differences in the concentrations of motile spermatozoa have been controlled by the application of Poisson distribution theory. Such variation has also been observed by Rogers et al. (1983) and is probably due to the influence of modulating factors such as stress, infection and, most importantly, ejaculation frequency (Rogers et al., 1983) on the penetrating potential of human spermatozoa. This inter-ejaculate variation in semen quality is a real phenomenon which must be considered when interpreting in-vitro fertilization data in a diagnostic context. The most practical solution to this problem is to recommend that the analysis is repeated on any sample scoring $<10 \%$ penetration during the initial assessment.

The ability of Poisson distribution theory to dissect the impact of sperm motility on the outcome of in-vitro fertilization experiments should be of value in both clinical and scientific studies of sperm function. Clinically, we have demonstrated that motility and the potential for fertilization are independent properties exhibited by spermatozoa, which may be differentially affected in cases of male infertility (Aitken, Ross \& Lees, 1983b; Aitken et al., 1983d). As a result, Poisson analysis should find application in a variety of clinical conditions associated with asthenozoospermia, such as oligozoospermia or varicocoele, in permitting analysis of the penetrating ability of the spermatozoa in isolation from the defects in motility. Similarly, such analyses will permit a more effective interpretation of in-vitro fertilization data obtained after treatments which disrupt sperm motility, including cryostorage, ingestion of potential contraceptive agents such as gossypol (Aitken et al., 1983a) and sulphasalazine (Toovey, Hudson, Hendry \& Levi, 1981), or in-vitro exposure to pharmacological reagents such as calmodulin inhibitors and adrenergic antagonists.

We gratefully acknowledge the technical assistance of Ms Fiona Best and the collaboration of Dr Martin Lees, Edinburgh Royal Infirmary, Dr Allan Templeton, Department of Obstetrics and Gynaecology, and Mr David Richardson, MRC Unit of Reproductive Biology, in the realization of these studies.

\section{References}

Aitken, R.J., Best, F.S.M., Richardson, D.W., Djahanbakhch, O. \& Lees, M.M. (1982a) The correlates of fertilizing capacity in normal fertile men. Fert. Steril. 38, 68-76.

Aitken, R.J., Best, F.S.M., Richardson, D.W., Djahanbakhch, O., Mortimer, D., Templeton, A.A. \& Lees, M.M. (1982b) An analysis of sperm function in cases of unexplained infertility: conventional criteria, movement characteristics and fertilizing capacity. Fert. Steril. 38, 212-221.

Aitken, R.J., Best, F.S.M., Richardson, D.W., Djahanbakhch, O., Templeton, A.A. \& Lees, M.M. (1982c) An analysis of semen quality and sperm function in cases of oligozoospermia. Fert. Steril. 38, 705-711.

Aitken, R.J., Liu, J., Best, F.S.M. \& Richardson, D.W. (1983a) An analysis of the direct effects of gossypol on human spermatozoa. Int. J. Androl. 6, 157-167.

Aitken, R.J., Ross, A. \& Lees, M.M. (1983b) Analysis of sperm function in Kartagener's Syndrome. Fert. Steril. 40, 696-698.

Aitken, R.J., Wang, Y.F., Liu, J., Best, F.S.M. \& Richardson, D.W. (1983c) The influence of medium composition, osmolarity and albumin content on the acrosome reaction and fertilizing capacity of human spermatozoa : development of an improved zona free hamster egg penetration test. Int. J. Androl. 6, 180193.

Aitken, R.J., Warner, P., Best, F.S.M., Templeton, A.A., Djahanbakhch, O., Mortimer, D. \& Lees, M.M. (1983d) The predictability of subnormal penetrating capacity in cases of unexplained infertility. Int. $J$. Androl. 6, 212-220.

Aitken, R.J., Best, F.S.M., Wamer, P. \& Templeton, A.A. (1984) A prospective study of the relationship between semen quality and fertility in cases of unexplained infertility: conventional semen profile, movement characteristics and zona free hamster egg penetration test. J. Androl. (in press).

Binor, Z., Sokoloski, J.E. \& Wolf, D.P. (1980) Penetration of the zona free hamster egg by human sperm. Fert. Steril. 33, 321-327.

Binor, Z., Sokoloski, J.E. \& Wolf, D.P. (1982) Sperm interaction with the zona free hamster egg. $J$. exp. Zool. 222, 187-193.

Cohen, J., Weber, R.R.A., Van der Vijver, J.C.M. \& Zeilmaker, G.H. (1982) In vitro fertilizing capacity of 
human spermatozoa with the use of zona free hamster ova: interassay variation and prognostic value. Fert. Steril. 37, 565-572.

Documenta Geigy Scientific Tables (1968) (6th edn). Geigy (UK), Macclesfield.

GLIM Manual (1978) Numerical Algorithms Group, Oxford.

Nelder, J.A. \& Wedderburn, R.W.M. (1972) Generalized linear models. Jl R. statist. Soc., Ser. A. 135, 370-385.

Richardson, D.W., Aitken, R.J. \& Loudon, N.B. (1984) The functional competence of human spermatozoa recovered following vasectomy. J. Reprod. Fert. 70, 575-579.

Rogers, B.J., van Campen, H., Ueno, M., Lambert, H., Bronson, R. \& Hale, R. (1979) Analysis of human spermatozoal fertilizing ability using zona-free ova. Fert. Steril. 32, 664-670.

Rogers, B.J., McCarville, C., Mygatt, G., Soderdahk, D. \& Hale, R. (1980) The use of in vitro fertilization for monitoring changes in human spermatozoal fertilizing ability associated with repair of varicocoele. Fert. Steril. 34, 311, Abstr.

Rogers, B.J., Perreault, S.D., Bentwood, B.J., McCarville, C., Hale, R.W. \& Soderdahl, D.W. (1983)
Variability in the human-hamster in vitro assay for fertility evaluation. Fert. Steril. 39, 204-211.

Stenchever, M.A., Spadoni, L.R., Smith, W.D., Karp, L.E., Shy, K.K., Moore, D.E. \& Berger, R. (1982) Benefits of the sperm (hamster ova) penetration assay in the evaluation of the infertile couple. Am. J. Obstet. Gynec. 143, 91-96.

Toovey, S., Hudson, E., Hendry, W.F. \& Levi, A.J. (1981) Sulphasalazine and male infertility: reversibility and possible mechanism. Gut 22, 445-451.

Wetherill, G.B. (1981) Intermediate Statistical Methods. Chapman and Hall, London.

World Health Organization (1980) Laboratory Manual for the Examination of Human Semen and Semen-Cervical Mucus Interaction. Eds M. A. Belsey, R. Eliasson, A. J. Galegos, K. S. Moghissi, C. A. Paulsen \& M. R. N. Prasad. Press Concern, Singapore.

Yanagimachi, R., Yanagimachi, H. \& Rogers, B.J. (1976) The use of zona-free animal ova as a test system for the assessment of fertilizing capacity of human spermatozoa. Biol. Reprod. 15, 471-476.

Received 19 December 1983 